\title{
Finite Element Modelling of Electrical Overhead Line Cables under Turbulent Wind Load
}

\author{
Dominik Stengel and Milad Mehdianpour \\ BAM Federal Institute for Materials Research and Testing, Unter den Eichen 87, 12205 Berlin, Germany \\ Correspondence should be addressed to Dominik Stengel; dominik.stengel@bam.de
}

Received 29 November 2013; Revised 28 January 2014; Accepted 14 February 2014; Published 27 March 2014

Academic Editor: Lucio Nobile

Copyright (C) 2014 D. Stengel and M. Mehdianpour. This is an open access article distributed under the Creative Commons Attribution License, which permits unrestricted use, distribution, and reproduction in any medium, provided the original work is properly cited.

\begin{abstract}
This paper presents a finite element model of an overhead transmission line using so called cable elements which allow reproducing the cable's nonlinear characteristics accurately employing only a few elements. Aerodynamic damping is considered in the equation of motion by taking into account the relative velocity between the flow of the wind and the moving structure. The wind flow itself is simulated by wave superposition making necessary assumptions on the lateral correlation between the wind velocities along the cable length. As result from the simulation, the following conclusions can be drawn. The first natural frequency of generally used wide spanning cables lies well below $1 \mathrm{~Hz}$ where also most of the energy content of the wind excitation is to be expected. Aerodynamic damping is significant for the moving cables holding very low structural damping which leads to a suppression of resonant amplification. This is particularly of interest regarding the support reaction which is dominated by the mean value and the so called background response. The latter is mostly influenced by the randomness of the wind flow, especially lateral to the main wind direction.
\end{abstract}

\section{Introduction}

Spanning over a few hundred meters, being light, and having slender structures, the wind acting on the cables contributes significantly to the overall loading of the suspension towers [1]. Both modelling of the cables and simulation of the acting wind field have to be undertaken with care to account for the particularities of such a horizontally expanded structure.

Such specific features in modelling involve the nonlinear structure, the nonlinear equation of motion, and an adequate simulation of the acting wind. The last is more often described in its nature along and vertically to the main wind direction than in its lateral character which is more important for horizontally expanded structures.

Many works have already dealt with this issue with different approaches, aims, and results. We start with early works [2] which gave a preliminary insight into the structural characteristics of overhead transmission line cables more than into the details of the wind excitation. Those works were soon followed by simulations [3] highlighting the importance of incorporating nonlinear effects and aerodynamic damping in regard to supporting reaction of suspended cables but without emphasizing the importance of the assumptions of the acting wind. Even in recent work, that aspect is not always included $[4,5]$. Later studies on wind tunnel models [6] stress again the need of detailed analysis when it comes to in-depth investigations of such a complex issue as the random wind excitation of nonlinear structures. Recent works have already shown that coherence of the acting wind has an impact on the response of sagging cables [7].

In due consideration of previous findings, this work presents an adequate procedure for finite element modelling of sagging cables as used in overhead transmission lines. In the nonlinear equation of motion, aerodynamic damping is implicitly considered by including the relative velocity between structure and wind flow and its impact on the characteristic of the resulting system's response is highlighted. Further, the generation of an adequate three-dimensional wind field is demonstrated and the importance of coherence is highlighted. The resulting reaction of the cable is analysed in regard of the support reaction. 


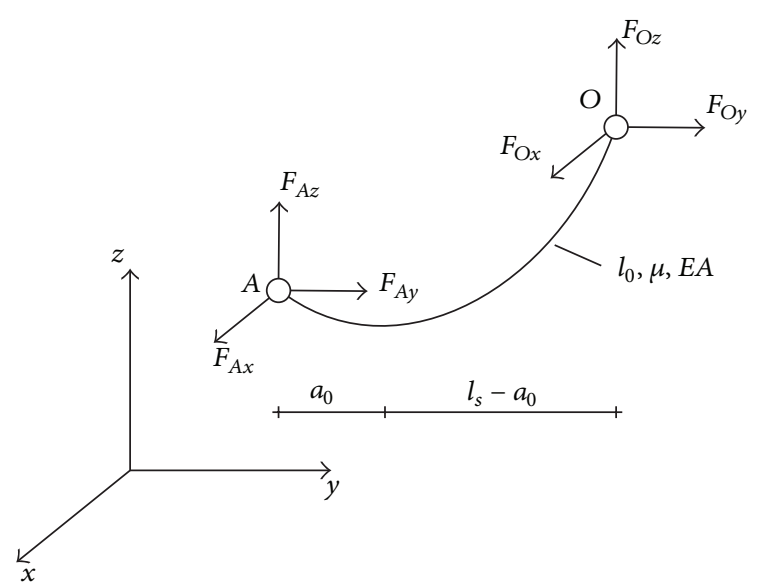

Figure 1: Three-dimensional cable element.

\section{Nonlinear Finite Element Formulations}

For modelling the cables of overhead transmission lines it is very useful to employ cable elements instead of truss elements. It can be shown that an accurate solution of the static catenary can be achieved with much less cable elements than with truss elements [8] since the catenary shape is already taken into consideration by the element shape per se.

Given the fact that the unstressed cable length $l_{0}$ of the general cable configuration is larger than the span length $l$ of the installed cable, it is the first step to determine the parameter out of the horizontal tension $H$ or stress $\sigma_{0}$, which is a set parameter in the construction of transmission line cables since it is a direct measure of the sag of the cable [9]:

$$
\begin{aligned}
l_{0}=l-\frac{\sigma_{0}^{2}}{8 E A \mu g} \times[ & \sinh \frac{2 \mu g\left(l_{s}-a_{0}\right)}{H} \\
& \left.+\sinh \frac{2 \mu g a_{0}}{H}+\frac{4 \mu g l_{s}}{H}\right] .
\end{aligned}
$$

Further cable parameters are the specific weight per unit length $\mu$ and the stiffness $E A$, with $g$ being the acceleration of gravity. The geometric parameters are the horizontal span length $l_{s}$ and the position of the lowest point $a_{0}$ as also given in Figure 1. The stressed length of the cable $l$ can be derived out of the equation of the catenary:

$$
l=\frac{H}{\mu g}\left[\sinh \frac{\mu g\left(l_{s}-a_{0}\right)}{H}+\sinh \frac{\mu g a_{0}}{H}\right] .
$$

On the cut-out element between the nodes $A$ and $O$ one can formulate the element mass and stiffness matrix out of the equilibrium of internal forces at the nodes [10]. Those are obtained by differentiating the axial tension $T$ over the Lagrangian coordinates $p$ and $s$ :

$$
\begin{gathered}
T\left(\frac{d x}{d p}\right)=-F_{A x}, \\
T\left(\frac{d y}{d p}\right)=-F_{A y}, \\
T\left(\frac{d z}{d p}\right)=-F_{A z}+\mu g s .
\end{gathered}
$$

Adopting Hooke's law for the relation between the axial tension and the strain $T=E A \epsilon$, the catenary coordinates can be transformed into the cartesians. By application of the boundary conditions and derivation which can be found in detail in [11], one can obtain the flexibility matrix $\left[F_{e}\right]$ and the stiffness matrix $\left[K_{e}\right]$ as the inverse of it:

$$
\begin{aligned}
\left\{\begin{array}{l}
d l_{x} \\
d l_{y} \\
d l_{z}
\end{array}\right\}= & {\left[\begin{array}{lll}
f_{11} & f_{12} & f_{13} \\
f_{21} & f_{22} & f_{23} \\
f_{31} & f_{32} & f_{33}
\end{array}\right]\left\{\begin{array}{l}
d F_{A x} \\
d F_{A y} \\
d F_{A z}
\end{array}\right\}=\left[F_{e}\right]\left\{\begin{array}{l}
d F_{A x} \\
d F_{A y} \\
d F_{A z}
\end{array}\right\}, } \\
f_{11}= & -\left(\frac{l_{0}}{E A}+\frac{1}{\mu g} \ln \left(\frac{T_{O}+F_{\mathrm{O} z}}{T_{A}-F_{A z}}\right)\right) \\
& +\frac{F_{A y}^{2}}{\mu g}\left(\frac{1}{T_{O}\left(T_{A}-F_{A z}\right)}-\frac{1}{T_{O}\left(T_{O}+F_{\mathrm{O} z}\right)}\right), \\
f_{12}=f_{21}= & \frac{F_{A y} F_{A x}}{w}\left(\frac{1}{T_{A}\left(T_{A}-F_{A z}\right)}-\frac{1}{T_{O}\left(T_{O}+F_{\mathrm{O} z}\right)}\right), \\
f_{22}= & -\left(\frac{l_{0}}{E A}+\frac{1}{\mu g} \ln \left(\frac{T_{O}+F_{\mathrm{O} z}}{T_{A}-F_{A z}}\right)\right) \\
+ & \frac{F_{A x}^{2}}{\mu g}\left(\frac{1}{T_{A}\left(T_{A}-F_{A x}\right)}-\frac{F_{A y}}{T_{A}\left(T_{O}+F_{\mathrm{O} z}\right)}\right), \\
& f_{23}=f_{32}=\frac{F_{A x}}{\mu g}\left(\frac{1}{T_{O}}-\frac{1}{T_{A}}\right), \\
& f_{33}=-\frac{l_{0}}{E A}-\frac{1}{\mu g}\left(\frac{F_{\mathrm{O} z}}{T_{O}}+\frac{F_{A z}}{T_{A}}\right) .
\end{aligned}
$$

The flexibility matrix is found iteratively until the boundary conditions for the element nodes are satisfied. The internal force vector contains the nodal forces $\left[R_{e}\right]=$ $\left[\begin{array}{llllll}F_{A x} & F_{A y} & F_{A z} & F_{\mathrm{O} x} & F_{\mathrm{O} y} & F_{\mathrm{Oz}}\end{array}\right]^{T}$ which are found iteratively until an equilibrium between given unstressed cable length $l_{0}$ and cable tension $T_{A}$ and $T_{O}$ at element nodes $A$ and $O$ is found:

$$
\begin{gathered}
T_{A}=\sqrt{F_{A x}^{2}+F_{A y}^{2}+F_{A z}^{2}}, \\
T_{O}=\sqrt{F_{O x}^{2}+F_{\mathrm{O} y}^{2}+F_{\mathrm{O} z}^{2}}, \\
F_{\mathrm{O} x}=-F_{A x}, \quad F_{\mathrm{O} y}=-F_{A y}, \quad F_{\mathrm{O} z}=-F_{A z}+\mu g l_{0} .
\end{gathered}
$$


TABLE 1: System parameters for the bundle conductor.

\begin{tabular}{|c|c|c|c|c|c|c|c|}
\hline $\begin{array}{l}\text { System } \\
\text { number }\end{array}$ & $\begin{array}{c}\text { Span length, } \\
l_{s}(\mathrm{~m})\end{array}$ & $\begin{array}{c}\text { Elastic } \\
\text { modulus, } E \\
\left(\mathrm{~N} / \mathrm{mm}^{2}\right)\end{array}$ & $\begin{array}{c}\text { Bundle } \\
\text { diameter, } d \\
(\mathrm{~mm})\end{array}$ & $\begin{array}{c}\text { Bundle } \\
\text { cross-section, } \\
A\left(\mathrm{~mm}^{2}\right)\end{array}$ & $\begin{array}{l}\text { Weight, } \mu \\
(\mathrm{kg} / \mathrm{m})\end{array}$ & Sag, $s(\mathrm{~m})$ & $\begin{array}{c}\text { Irvine's } \\
\text { parameter, } \lambda^{2}\end{array}$ \\
\hline 1 & & & & & & - & $\rightarrow 0$, string \\
\hline 2 & & & & & & 6 & 10 \\
\hline 3 & 400 & 74000 & $4 \cdot 22.4$ & $4 \cdot 297.7$ & $4 \cdot 0.998$ & 13 & 100 \\
\hline 4 & & & & & & 19 & 300 \\
\hline 5 & & & & & & - & $\rightarrow \infty$ \\
\hline
\end{tabular}

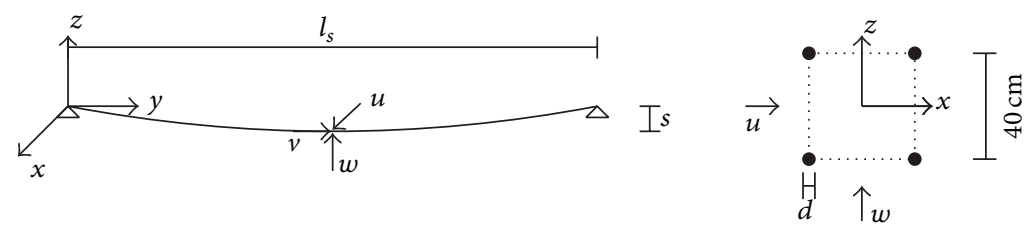

FIGURE 2: Presentation of line and cross-section.

The mass is assumed to be uniformly distributed along the element which gives a simple formulation of the mass matrix $\left[M_{e}\right]$ :

$$
\left[M_{e}\right]=\frac{\mu l_{0}}{2}\left[\begin{array}{lll}
1 & 0 & 0 \\
0 & 1 & 0 \\
0 & 0 & 1
\end{array}\right] .
$$

Once obtained for each element, the total flexibility or rather stiffness matrix and internal force vector $\{R\}$ is obtained by assembling the element matrices [12].

The static equilibrium position is then found by applying the Newton-Raphson method which iteratively varies the nodal displacement until the internal forces at the nodes are in equilibrium.

\section{Model Description}

The procedure is used to model a single span of a transmission line cable of a typical configuration as given in Table 1 and displayed in Figure 2. A bundle conductor of four stranded wires of aluminium and steel Al/St 265/35 is chosen to exemplary evaluate the specific nonlinear characteristics of common electrical overhead cables.

The model is assembled by 50 elements which allow estimating the first 6 modes of vibration accurately. The unstressed cable length $l_{0}$ or the sag in midspan as its effect is kept variable to investigate its influence on the dynamic properties of the system. All that information can be combined in the so called Irvine's parameter $\lambda^{2}$ :

$$
\lambda^{2}=\frac{\left(\mu g l_{s} / H\right)^{2} l_{s}}{H l / E A} .
$$

\section{Modal Analysis}

Stranded wires as used for the cables possess very low structural damping. So the eigenvalues $\omega^{2}$ and eigenvectors $\{q\}=\left\{\begin{array}{lll}q_{x} & q_{y} & q_{z}\end{array}\right\}^{T}$ of the system under dead load can be found by linearization of the equation of motion around the static solution:

$$
\left([K]-\omega^{2}[M]\right)\{q\}=0 .
$$

The out-of-plane modes are the swaying modes of the whole span with pendulum frequencies $\omega_{n}=n \pi / l_{s} \sqrt{H / \mu}$. For in-plane modes the nonlinear character of the cables gets more significant. Table 2 summarizes the results for the inplane modes for different models of cables, starting from the theory of a perfect string on the left over, an augmentation of Irvine's parameter to the theory of an inextensible cable with large values of $\lambda^{2}$ on the right.

For small values of $\lambda^{2}$ the system resembles the linear system of a string with eigenfrequencies equal to the outof-plane ones. With augmenting $\lambda^{2}$ one can observe a so called modal crossing, resulting in an irregular occurrence of symmetric $(s)$ and antisymmetric $(a)$ modes. For $\lambda^{2}=300$ the system can again be linearized with an analytical solution for the eigenfrequencies [13]. The solutions for antisymmetric modes are equal; the solutions for symmetric modes are almost similar to those obtained for $\lambda^{2} \rightarrow \infty$ according to the following, which are given in Table 2 in the last row for comparison:

$$
\begin{gathered}
\omega_{n, a}=\frac{2 \pi n}{l_{s}} \sqrt{\frac{H}{\mu}}, \\
\omega_{1, s}=\frac{2.86 \pi}{l_{s}} \sqrt{\frac{H}{\mu}}, \quad \omega_{2, s}=\frac{4.92 \pi}{l_{s}} \sqrt{\frac{H}{\mu}}, \\
\omega_{n, s}=\frac{(2 n+1) \pi}{l_{s}} \sqrt{\frac{H}{\mu}} .
\end{gathered}
$$

The accordance between simulation and theory as observable from the last two rows of Table 2 confirms the application of cable elements to model the dynamic behaviour of transmission line cables. 
TABle 2: Antisymmetric (a) and symmetric (s) in-plane modes for different parameters of $\lambda^{2}$.

\begin{tabular}{|c|c|c|c|c|c|c|c|c|c|c|c|}
\hline \multirow{2}{*}{$\begin{array}{l}\text { System } \\
\text { Mode }\end{array}$} & & \multicolumn{2}{|c|}{ Number 1} & \multicolumn{2}{|c|}{ Number 2} & \multicolumn{2}{|c|}{ Number 3} & \multicolumn{2}{|c|}{ Number 4} & \multicolumn{2}{|c|}{ Number 5 [13] } \\
\hline & & a & $\mathrm{s}$ & $\mathrm{a}$ & $\mathrm{s}$ & $\mathrm{a}$ & $\mathrm{s}$ & $\mathrm{a}$ & $\mathrm{s}$ & $\mathrm{a}$ & $\mathrm{s}$ \\
\hline \multirow{6}{*}{$\begin{array}{l}\text { Angular frequency } \\
(\mathrm{rad} / \mathrm{s})\end{array}$} & $\omega_{1}$ & & 1.42 & & 1.90 & 1.91 & & 1.57 & & 1.59 & \\
\hline & $\omega_{2}$ & 2.84 & & 2.85 & & & 2.50 & & 2.23 & & 2.25 \\
\hline & $\omega_{3}$ & & 4.26 & & 4.31 & & 3.35 & 3.16 & & 3.14 & \\
\hline & $\omega_{4}$ & 5.68 & & 5.71 & & 3.82 & 4.83 & & 3.74 & & 3.86 \\
\hline & $\omega_{5}$ & & 7.10 & & 7.14 & 5.73 & & 4.74 & & 4.71 & \\
\hline & $\omega_{6}$ & 8.52 & & 9.98 & & & 6.70 & & 5.60 & & 5.50 \\
\hline
\end{tabular}

\section{Nonlinear Equation of Motion}

Deformation due to static and dynamic loading $\{F\}$, the stiffness $[K]$, and the internal force $\{R\}$ has to be updated. In each time step $i$, the system matrices are found by iteration over $k$. Again structural damping is neglected. The solution of that nonlinear equation of motion is found with the Houbolt Method [12]:

$$
[M]\left\{\ddot{q}_{i}^{k}\right\}+\left[K_{i}^{k}\right]\left\{\Delta q^{k}\right\}=\left\{F_{i}\left(\left\{\dot{q}_{i}^{k}\right\}\right)\right\}-\left\{R_{i}^{k}\right\} .
$$

The external force is given as a function of the system's velocity $\{\dot{q}\}$. That implies that there are self-induced forces due to the movement of the cable resulting in aerodynamic damping. That effect will be visualized by simulating the free oscillation.

The bundle conductor is considered as a single cable, neglecting the effect of bundle rotation and lifting forces. Such effects could easily be included but are not part of this study since they would request enhanced wind tunnel tests.

Wind force is applied under quasi-steady assumption which means that the resulting forces are determined by the instantaneous wind velocity acting on the cable. Other dynamic excitations are neglected, such as vortex shedding, which is supported by vortex-shedding frequencies $f_{w}$ much higher than the first natural frequencies of the conductor, assuming a Strouhal number St $=0.2[14]$ :

$$
f_{w}=\operatorname{St} \frac{u}{d} \text {. }
$$

Consequently, wind forces are determined by air density $\rho=1.25 \mathrm{~kg} / \mathrm{m}^{2}$, the aerodynamic force coefficient $c_{f}=1.0$ [15], diameter $d$, length $l$, the relative velocity between acting flow $u_{i}$ and $w_{i}$ in $x$ and $z$ direction, respectively, and structure:

$$
\begin{aligned}
& F_{x i}=\frac{\rho}{2} c_{f} d l\left(u_{i}-\dot{q}_{x i}\right)^{2}, \\
& F_{z i}=\frac{\rho}{2} c_{f} d l\left(w_{i}-\dot{q}_{z i}\right)^{2} .
\end{aligned}
$$

Even with wind velocity $u=0$ and $w=0$, there is always a force acting on the cable opposite to the direction of movement. To illustrate the effect, the before presented cable is deflected statically and out of the cable plane and left free to oscillate. Time step is set to $\Delta t=0.04 \mathrm{~s}$.

As one can observe in Figure 3 there is a significant effect of damping especially during the first few periods. That

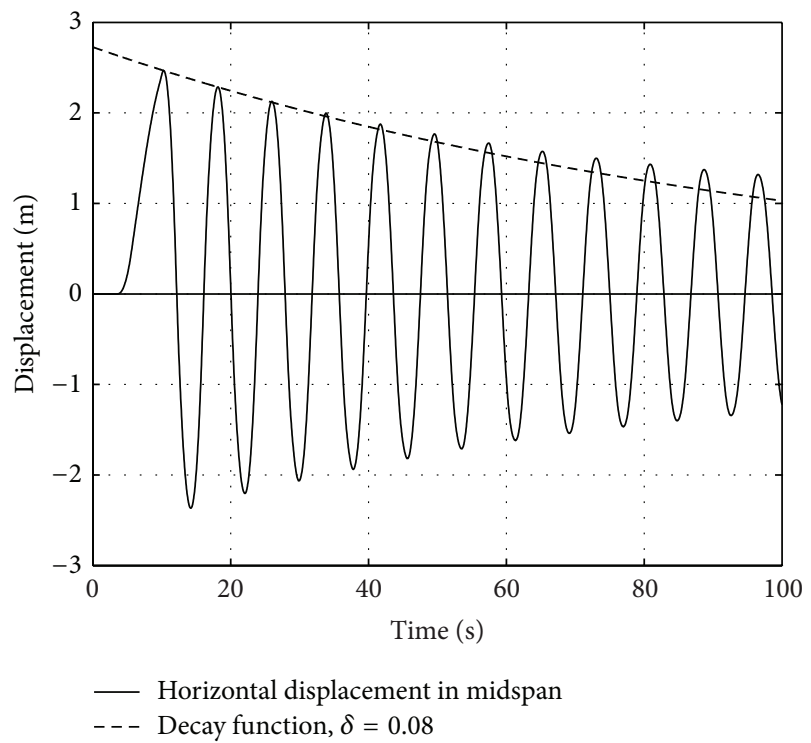

FIGURE 3: Oscillation of cable.

effect diminishes when the movement gets less and with it the system's velocity. For comparison, a decay curve with decay factor $\delta=0.08$ is also displayed. In comparison to structural damping assumed for stranded wires [16], the resulting aerodynamic damping is of about one order of magnitude larger and is to be expected much larger with increasing wind velocity since it goes along with the term $2 u_{i} \dot{q}_{x i}$.

\section{Generation of a Three-Dimensional Wind Field}

The wind field is composed of three velocity components $u$, $v$, and $w$ acting in the three dimensions of the system $x, y$, and $z$, respectively. The component $u$ is defined as the main wind direction with mean wind velocity $\bar{u}$. Wind parallel to the line direction, $v$, is considered ineffective for the system's response.

Based on general assumptions on the statistical characteristics of wind velocities, a three-dimensional field of time histories of wind velocities perpendicular to the cable's axial 
direction is generated with the components of turbulence $u^{\prime}$ and $w^{\prime}$ dependent on time and location:

$$
u(x, y, z, t)=\bar{u}(z)+u^{\prime}(x, y, z, t) .
$$

Mean wind velocities $\bar{u}$ are determined dependent on the height above ground $z$, with a reference wind speed at $10 \mathrm{~m}$, height $v_{b}=25 \mathrm{~m} / \mathrm{s}$, and terrain roughness length $z_{0}=0.05$. The ground level is assumed as a horizontal line $40 \mathrm{~m}$ beyond the cable supports:

$$
\bar{u}(z)=0.19 v_{b} \ln \left(\frac{z}{z_{0}}\right) .
$$

Cross-correlations of the turbulence components are neglected. This simplification is justified by a negligible influence of the vertical wind component on the system's response and a considerable reduction in computational effort:

$$
S(f)=\left[\begin{array}{cc}
S_{u} & S_{u w} \\
S_{w u} & S_{w}
\end{array}\right] \approx\left[\begin{array}{cc}
S_{u} & 0 \\
0 & S_{w}
\end{array}\right] .
$$

The independent spectral density matrices $S_{u}$ and $S_{w}$ are composed by the auto- and cross-correlations of the nodes 1 to $n$ :

$$
S_{u}(f)=\left[\begin{array}{cccc}
S_{u_{1} u_{1}} & S_{u_{1} u_{2}} & \cdots & S_{u_{1} u_{n}} \\
S_{u_{2} u_{1}} & S_{u_{2} u_{2}} & \cdots & S_{u_{2} u_{n}} \\
\vdots & \vdots & \ddots & \vdots \\
S_{u_{n} u_{1}} & S_{u_{n} u_{2}} & \cdots & S_{u_{n} u_{n}}
\end{array}\right]
$$

For generating the fluctuating wind velocities a method of wave superposition [17] is chosen. They are generated at the locations of the nodes in the static equilibrium position. Due to the sag of the cable the nodes are in different heights which results in an initially two-dimensional wind field in the cable plane. The out-of-plane displacement is allowed by applying Taylor's hypothesis of frozen turbulence assuming that the fluctuating wind velocity $u^{\prime}$ at point $x+\Delta x$ and time $t$ are equal to the wind velocity at point $x$ and time $t-\Delta t$, with $\Delta t=\Delta x / \bar{u}:$

$$
u^{\prime}(x+\Delta x, t)=u^{\prime}\left(x, t-\frac{\Delta x}{\bar{u}}\right)
$$

For each time history of fluctuating wind velocity, the turbulence spectrum $S_{u}$ according to the von Kármán spectrum is assumed:

$$
\frac{f S_{u}(f)}{\sigma_{u}^{2}(z)}=\frac{4 f_{n}}{\left(1+70.8 f_{n}^{2}\right)^{5 / 6}}, \quad f_{n}=\frac{f L_{u x}(z)}{\bar{u}(z)} .
$$

Variance $\sigma_{u}^{2}$ and turbulence integral scale in $x$-direction $L_{u x}$ are determined according to European standard [18]:

$$
\begin{gathered}
\sigma_{u}=0.19 v_{b}, \\
L_{u x}=300\left(\frac{z}{200}\right)^{0.67+0.05 \ln z_{0}} .
\end{gathered}
$$

The time history is then generated by superposition of $k$ waves with frequency $f_{k}$ with a random phase angle $\phi_{k} \in$ $\left[\begin{array}{ll}0 & 2 \pi\end{array}\right]$ :

$$
u^{\prime}(t)=\sum_{k=1}^{N} \sqrt{2 S_{u u}\left(f_{k}\right) \Delta f} \cos \left(2 \pi f_{k} t+\phi_{k}\right) .
$$

Cross-correlation between the locally separated time histories is considered by establishing of a full spectral density matrix containing the auto-density function on the diagonal and the cross-density functions on the off diagonal positions. The cross-spectra are estimated by their relation via the coherence function $\gamma$ which can again be approximated by a simple formulation dependent on the decay constants $C_{u y}$ and $C_{u z}$ :

$$
\begin{aligned}
\gamma_{i j}(f) & =\frac{S_{i j}(f)}{\sqrt{S_{i i}(f) S_{j j}(f)}} \\
& =\exp \left(-\frac{2 f \sqrt{C_{u y}^{2} \Delta y^{2}+C_{u z}^{2} \Delta z^{2}}}{\bar{u}_{i}+\bar{u}_{j}}\right) .
\end{aligned}
$$

There are several suggestions on the decay constants which show a wide scatter over the evaluations [19]. Suggestions to adopt here need to meet with the requirements of the structure, which is wide spanning in horizontal direction. For the vertical direction $C_{u z}=11$ is chosen, whereas for the lateral direction the maximum of $C_{u y}=11$ and a formulation dependent on the distance $\Delta y$ and the mean height above ground $\bar{z}$ of the nodes are chosen [20]:

$$
C_{u y}=8.5\left(\frac{\Delta y}{\bar{z}}\right)^{0.25} \text {. }
$$

That definition of the lateral decay factor leads to less correlation of the wind time histories along the line. If the dynamic action is less correlated along the line, the system's excitation is hindered. Further attention has to be paid to the element length. The forces acting along the element are applied as a nodal force which postulates full correlation along the element length. That leads to an overestimation of fluctuating wind forces if the element length is too long [21]. The present division into 50 elements satisfies the given formula for maximum element length:

$$
l \leq \frac{2 \bar{u}}{C_{u y} f} .
$$

Figure 4 shows the example of one generated time history of 10 minutes as well as the correlation coefficient for all generated time histories. Time histories for the vertical component are generated accordingly assuming mean wind velocity $\bar{w}=0, \sigma_{w} / \sigma_{u}=0.5$, and $L_{w x} / L_{u x}=0.1$ [22].

\section{Cable Response to Turbulent Wind Excitation}

The cable response will be evaluated in terms of the support reaction. That again is divided into mean and fluctuating part 

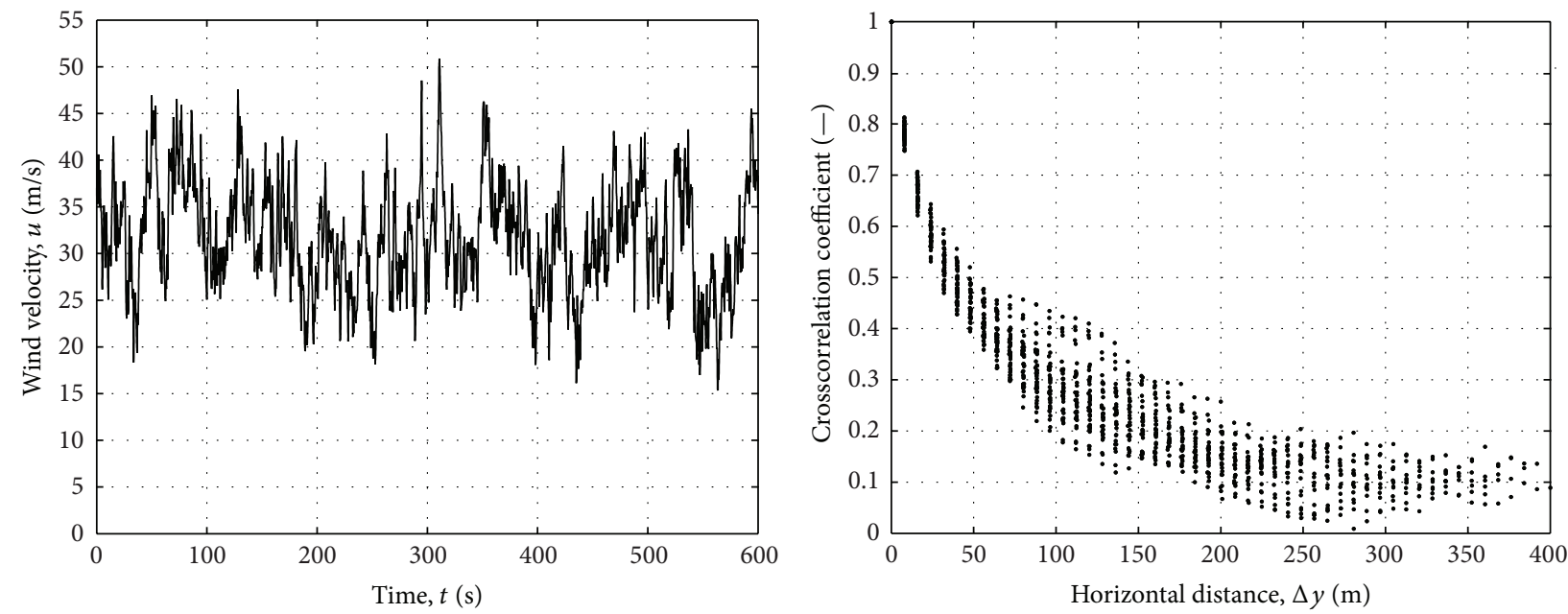

FIGURE 4: Example of generated time history of wind velocity and cross-correlation coefficient between generated time histories.

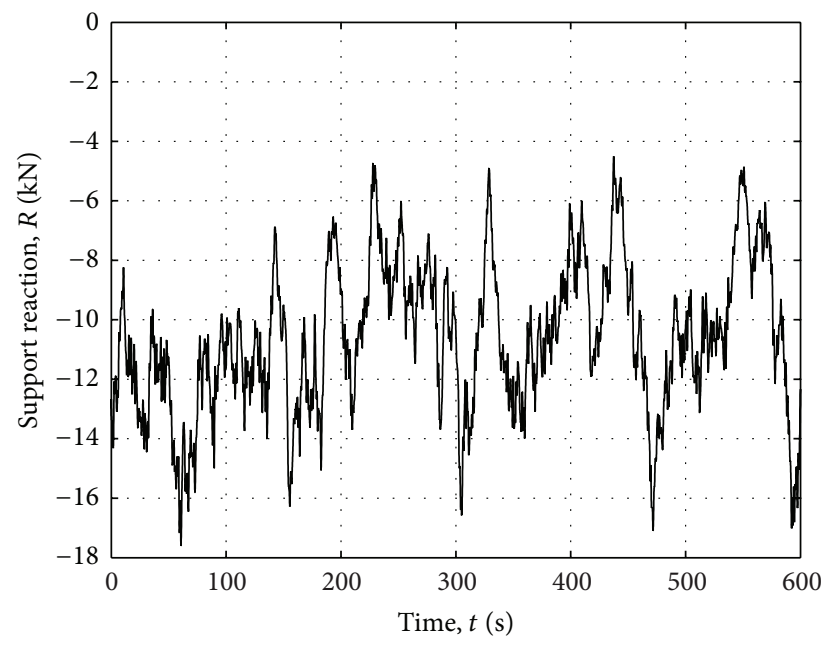

FIgURE 5: Time history of support reaction in wind direction.

since the peak response $\widehat{R}$ to be expected is defined by a so called peak factor $k_{p}[23]$ :

$$
\widehat{R}=\bar{R}+k_{p} \sigma_{R} .
$$

Figure 5 shows the time history of the support reaction in wind direction for wind velocities generated as mentioned above. It is obviously dominated by the mean part of the response which follows the general assumption being equal to half the mean wind force and only dependent on the mean wind velocity:

$$
\bar{R}=0.5 \bar{F}=0.5 \frac{\rho}{2} c_{f} d L \bar{u}^{2} .
$$

Regarding the fluctuating component of the system's response, there is almost no resonant amplification observable. That can best be shown by its spectral density function as in Figure 6 and is in accordance with observations on real structures [24]. For that reason, the fluctuating response is

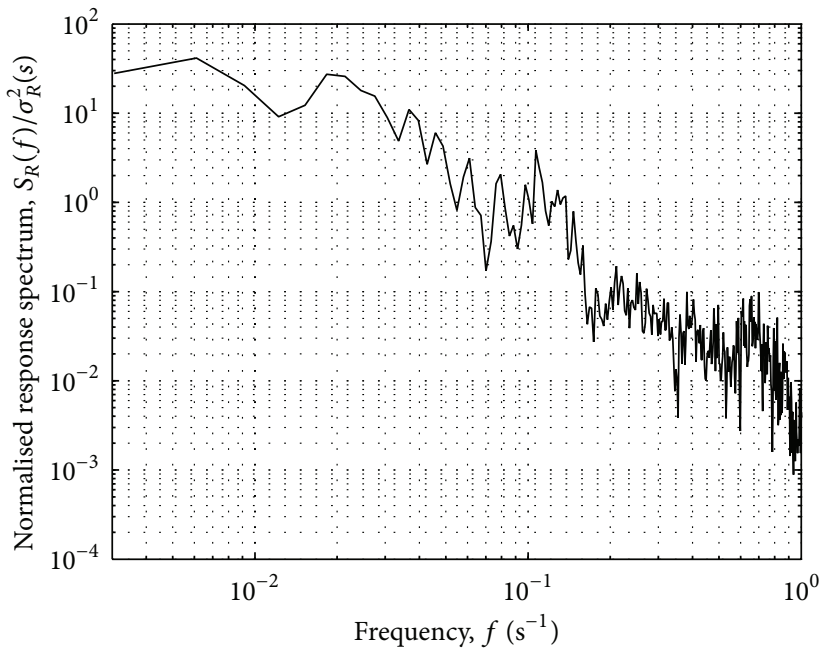

FIGURE 6: Normalised response spectrum of simulated support reaction.

particularly governed by the fluctuating action of wind with most energy content up to $1 \mathrm{~Hz}$.

The system is therefore sensible for the assumptions on the distribution of wind along the line, namely, its correlation, and it is obvious that those assumptions need to be handled with care, in case the results will be interpreted.

The peak factor in (24) can be estimated out of the probability distribution of the response. Generally a value between 3 and 4 is assumed [25], which corresponds to a normal distribution of the response. Despite the nonlinearities in system and equation of motion, the simulated system's response can be assumed as normal distributed, as shown in Figure 7.

Assuming normal distribution, the peak factor can be analytically determined by assuming independent peaks [26], where $v_{0}$ indicates the frequency of local maxima, $T$ indicates 


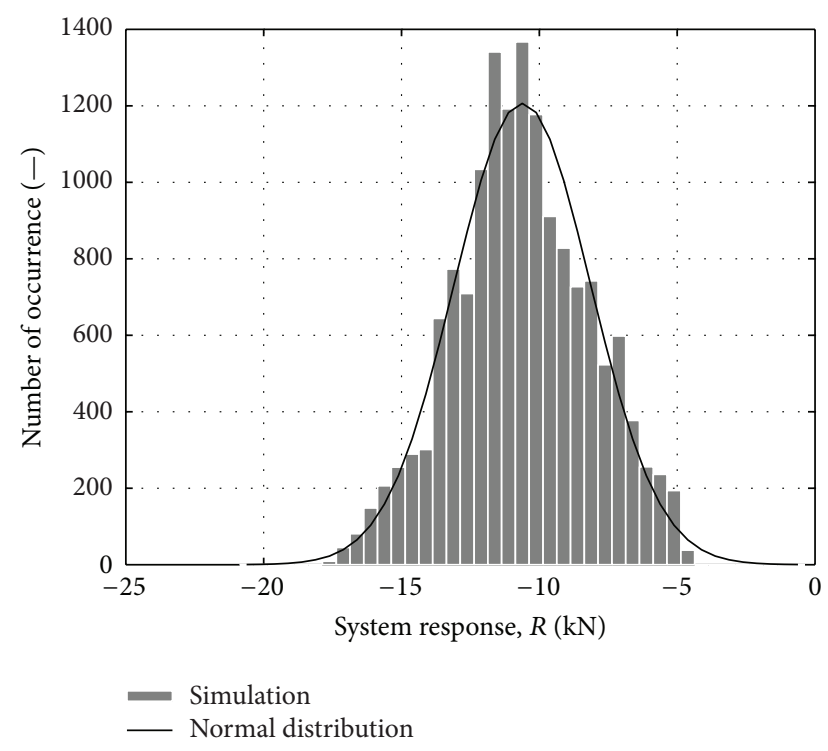

FIGURE 7: Frequency distribution of support reaction.

the duration of observation, and $e=0.577216$ is the EulerMascheroni's constant:

$$
k_{p}=\sqrt{2 \ln \left(v_{0} T\right)}+\frac{e}{\sqrt{2 \ln \left(v_{0} T\right)}} .
$$

Determining the peak factor from the time histories of the simulations by counting of positive zero crossings leads to values of approximately $k_{p}=3.5$. But that formulation is known to be conservative [27] and further investigations should be done ahead of drawing early conclusions on that topic. Furthermore, normal distribution is not self-evident and cannot be assumed for any transmission line system.

\section{Conclusions}

A complete model of a single span overhead transmission line cable was presented. Crucial parameters such as sources of nonlinearities of structure and motion have been highlighted and evaluated. Particular emphasis was drawn on the nonlinear equation of motion that includes the effect of aerodynamic damping which must not be neglected when dealing with structures of relatively low structural damping in comparison to aerodynamic damping.

Considering the effect of aerodynamic damping, the structural response highly depends on mean and fluctuating wind velocities. Great care has to be bestowed to the loading distribution along the line. Important parameters such as length scale and decay factors of wind turbulence which can be found in standards are generally defined for the usual application of building constructions with possible high vertical but only small horizontal extend. For the wide spanning transmission lines, more accurate assumptions need to be adopted and have been suggested in this work.

Estimation of the system's peak response requires assumptions on the statistical nature of the response. In this work, possible sources of errors which can have an impact on the characteristic of the probability distribution of the response have been presented. Only if the dynamic response is estimated with sufficient reliability, it is possible to draw conclusions on the characteristics of the random process of wind excitation which would allow estimating the system's peak response.

\section{Conflict of Interests}

The authors declare that there is no conflict of interests regarding the publication of this paper.

\section{Acknowledgment}

The authors gratefully acknowledge the sponsoring of the research project MOSYTRAF which constitutes the framework of the present study by the transmission system operators 50 Hertz Transmission GmbH, E.ON Netz GmbH, and TenneT TSO GmbH.

\section{References}

[1] R. Kadaba, "Response of electrical transmission line conductors to extreme wind using field data," in Civil Engineering, p. 142, Texas Tech University, 1988.

[2] C. Manuzio, "Wind effects on suspended cables," in Wind Effects on Buildings and Structures, pp. 337-370, University of Toronto Press, Ottawa, Canada, 1968.

[3] M. J. Matheson and J. D. Holmes, "Simulation of the dynamic response of transmission lines in strong winds," Engineering Structures, vol. 3, no. 2, pp. 105-110, 1981.

[4] A. Y. Shehata, A. A. El Damatty, and E. Savory, "Finite element modeling of transmission line under downburst wind loading," Finite Elements in Analysis and Design, vol. 42, no. 1, pp. 71-89, 2005.

[5] F. Cluni, V. Gusella, and F. Ubertini, "A parametric investigation of wind-induced cable fatigue," Engineering Structures, vol. 29, no. 11, pp. 3094-3105, 2007.

[6] A. M. Loredo-Souza and A. G. Davenport, "The effects of high winds on transmission lines," Journal of Wind Engineering and Industrial Aerodynamics, vol. 74-76, pp. 987-994, 1998.

[7] V. Gattulli, L. Martinelli, F. Perotti, and F. Vestroni, "Dynamics of suspended cables under turbulence loading: reduced models of wind field and mechanical system," Journal of Wind Engineering and Industrial Aerodynamics, vol. 95, no. 3, pp. 183-207, 2007.

[8] R. Karoumi, "Some modeling aspects in the nonlinear finite element analysis of cable supported bridges," Computers and Structures, vol. 71, no. 4, pp. 397-412, 1999.

[9] B. Yan, X. Lin, W. Luo, Z. Chen, and Z. Liu, "Numerical study on dynamic swing of suspension insulator string in overhead transmission line under wind load," IEEE Transactions on Power Delivery, vol. 25, no. 1, pp. 248-259, 2010.

[10] H. B. Jayaraman and W. C. Knudson, "A curved element for the analysis of cable structures," Computers and Structures, vol. 14, no. 3-4, pp. 325-333, 1981.

[11] H.-T. Thai and S.-E. Kim, "Nonlinear static and dynamic analysis of cable structures," Finite Elements in Analysis and Design, vol. 47, no. 3, pp. 237-246, 2011. 
[12] K. J. Bathe, Finite Element Procedures in Engineering Analysis, 1982.

[13] H. M. Irvine and T. K. Caughey, "The linear theory of free vibrations of a suspended cable," Proceedings of the Royal Society of London A, vol. 341, no. 1626, pp. 299-315, 1974.

[14] L. Carassale and G. Piccardo, "Non-linear discrete models for the stochastic analysis of cables in turbulent wind," International Journal of Non-Linear Mechanics, vol. 45, no. 3, pp. 219-231, 2010.

[15] IEC, Design Criteria of Overhead Transmission Lines, International Electrotechnical Commission, Geneva, Switzerland, 2003.

[16] M. J. Paluch, T. T. O. Cappellari, and J. D. Riera, "Experimental and numerical assessment of EPS wind action on long span transmission line conductors," Journal of Wind Engineering and Industrial Aerodynamics, vol. 95, no. 7, pp. 473-492, 2007.

[17] M. Shinozuka and C.-M. Jan, "Digital simulation of random processes and its applications," Journal of Sound and Vibration, vol. 25, no. 1, pp. 111-128, 1972.

[18] EN, Eurocode Actions on Structures-General Actions, in Part 14, vol. 1, Wind Actions, 2010.

[19] G. Solari, “Turbulence Modeling for Gust Loading," Journal of Structural Engineering, vol. 113, no. 7, pp. 1550-1569, 1987.

[20] G. Solari, "Gust buffeting. I: peak wind velocity and equivalent pressure," Journal of Structural Engineering, vol. 119, no. 2, pp. 365-382, 1993.

[21] V. Denoël, "Accounting for coherence in wind forces in finite element models," in Proceedings of the European Conference on Structural Dynamics (EURODYN '05), pp. 2219-2224, Millpress, 2005.

[22] G. Solari and G. Piccardo, "Probabilistic 3-D turbulence modeling for gust buffeting of structures," Probabilistic Engineering Mechanics, vol. 16, no. 1, pp. 73-86, 2001.

[23] A. G. Davenport, "Gust response factors for transmission line loading," in Proceedings of the 5th International Conference on Wind Engineering, Pergamon Press, Fort Collins, 1979.

[24] D. Stengel and M. Mehdianpour, "Wind measurements along a high-voltage overhead transmission line in Northern Germany," in Proceedings of the 4th International Conference on Integrity, Reliability \& Failure, INEGI-Instituto de Engenharia Mecânica e Gestao Industrial, Funchal, Portugal, 2013.

[25] A. G. Davenport, "How can we simplify and generalize wind loads?" Journal of Wind Engineering and Industrial Aerodynamics, vol. 54-55, pp. 657-669, 1995.

[26] A. Preumont, "On the peak factor of stationary Gaussian processes," Journal of Sound and Vibration, vol. 100, no. 1, pp. 15-34, 1985.

[27] C. Floris and L. De Iseppi, "The peak factor for gust loading: a review and some new proposals," Meccanica, vol. 33, no. 3, pp. 319-330, 1998. 

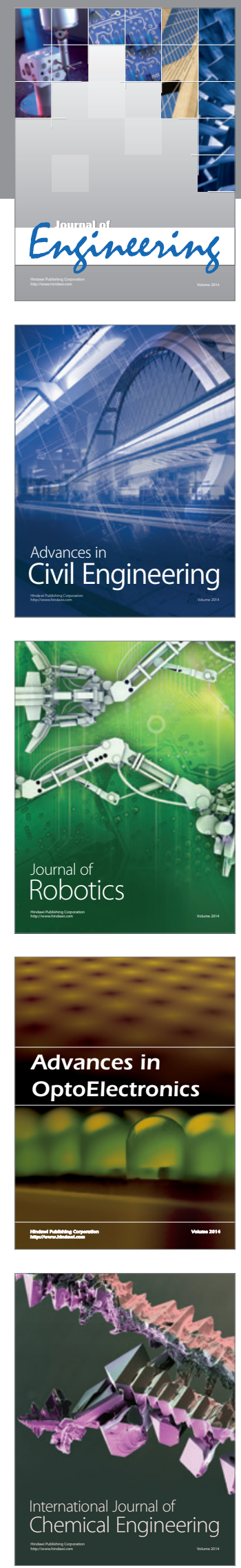

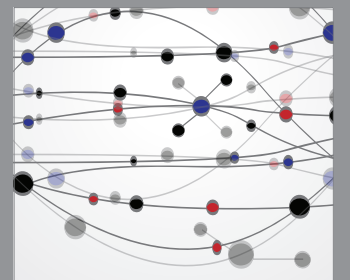

The Scientific World Journal
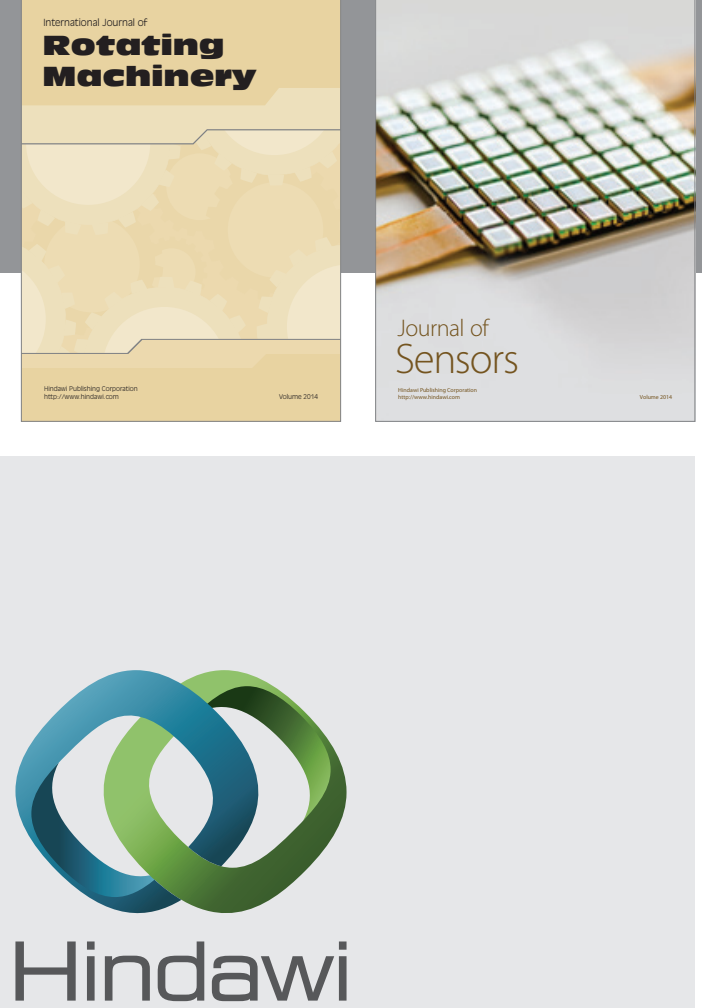

Submit your manuscripts at http://www.hindawi.com
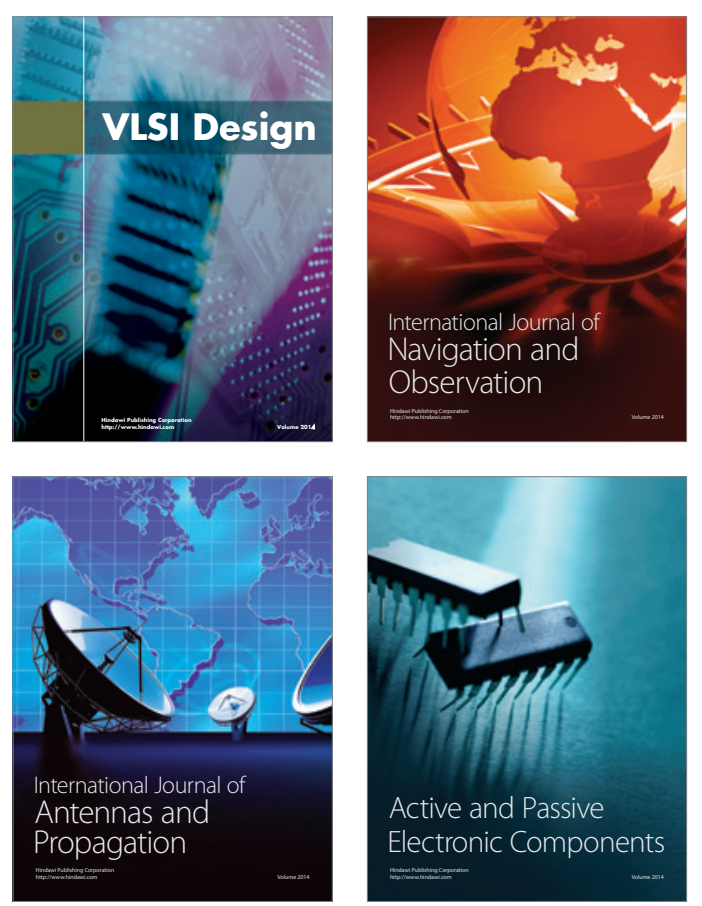
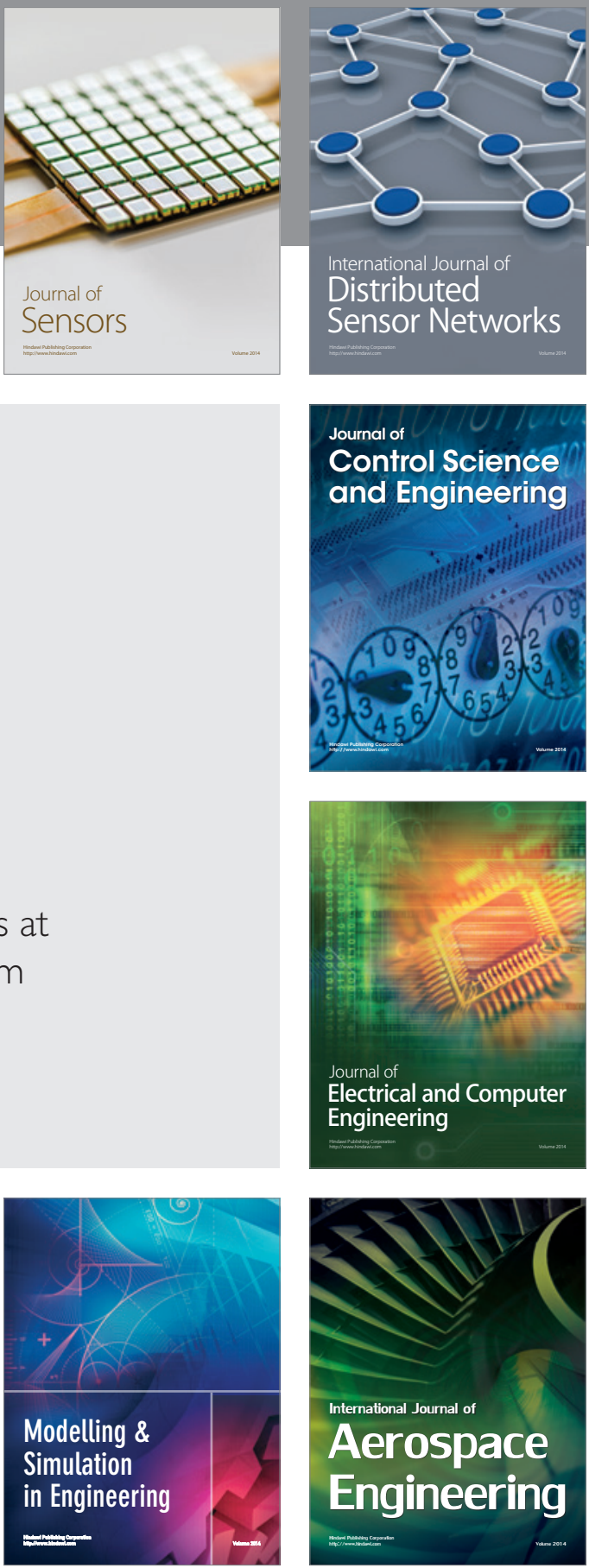

Journal of

Control Science

and Engineering
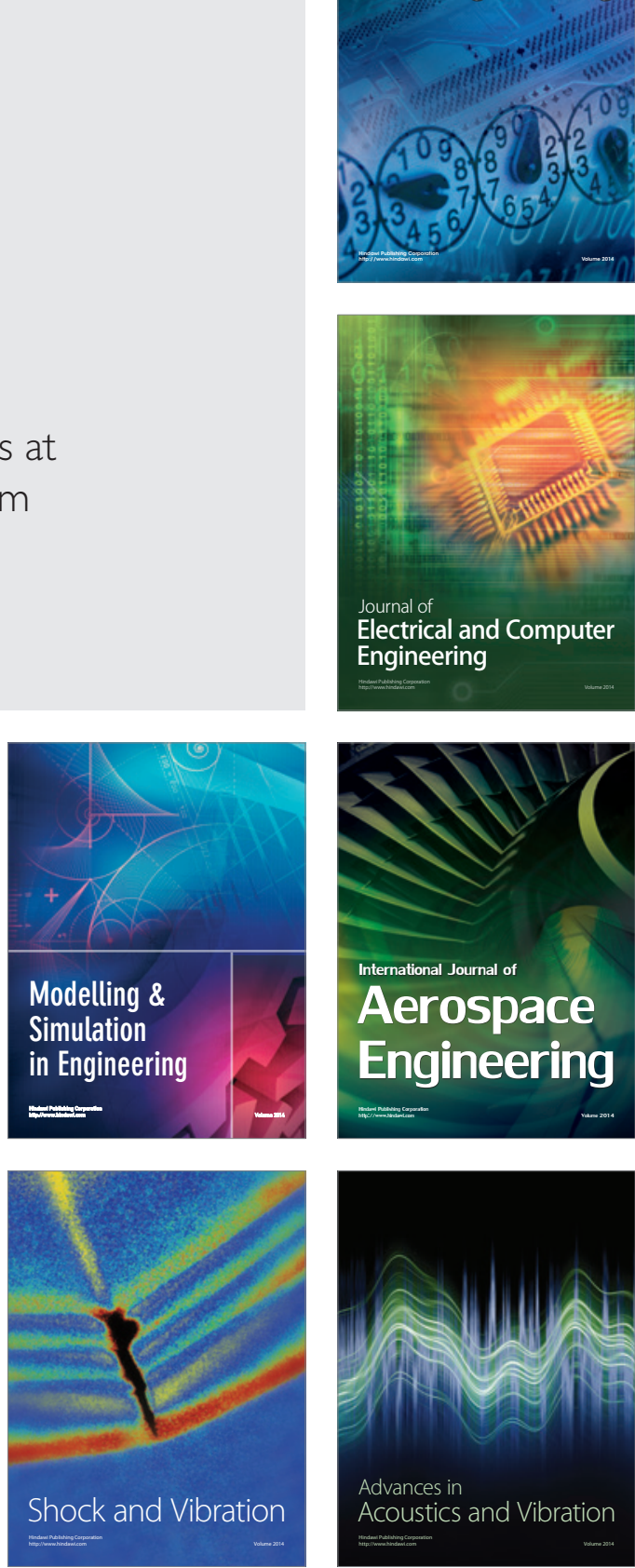